\title{
Feeling of agency versus judgment of agency in passive movements with various delays from the stimulus
}

\author{
Ignat A. Dubynin ${ }^{*}$, Sergei L. Shishkin \\ National Research Center "Kurchatov Institute", Moscow, Russia \\ *Corresponding author. Email: ignd@mail.ru
}

Background. The sense of agency (SoA) provides us with the experience of being a physical agent with free will. On a phenomenological basis, SoA can be divided into sensory components (feeling of agency, FoA) and more cognitive components (judgment of agency, JoA). Both these components can be independently measured.

Objective and Method. A new method was developed to test the possibility of preserving SoA and its components in the atypical conditions of passive movements. Parameters of the participant's movement in response to a visual stimulus (reaction time, speed, and amplitude) were measured and used to control a servo that simulated the movement (executed passive movements). The scores on the psychometric scale of the agency and the event-related potentials (ERPs) were recorded for variable movement delays relative to the stimulus onset.

Results. It was found that the FoA was not present under passive movement conditions. At the same time, participants associated these movements with their own activity (JoA), even when their delay after the stimulus onset was too short to be actively reproduced. The somatosensory ERPs' amplitude decreased for the expected movements, demonstrating an inverse relationship with the agency scores. The lowest amplitude was observed when movements were actuated by another hand. The results can be explained using a predictive forward model, since the FoA was not observed in the absence of active movements. On the other hand, the ERPs' data and the presence of JoA with various delays between the stimulus and movement support the postdictive model of agency, where the leading role is assigned to prejudice and contextual knowledge related to the action.

Conclusion. It seems that the "context pressure" of the situation, demanding a mandatory response to the stimulus, forms a cognitive prediction of movements without firm sensory representation.

Keywords: action, free will, mental chronometry, passive movement, feeling of agency, judgment of agency, sense of agency, somatosensory event-related potentials (ERPs) 


\section{Introduction}

The sense of agency (SoA) is a specific inner experience that provides us with the feeling of being a physical agent with free will, and with the foundation necessary for sensing the spatial aspects of our "I" in action. It is an important part of human consciousness, forming the fundamental aspect of self-awareness (Gallagher, 2002).

There are several theoretical concepts that explain the emergence of SoA. One explanation is based on an internal feed-forward model, according to which motor commands and predictions about their sensory effect in a successful situation are stored in the memory (Blakemore, Wolpert, \& Frith, 2000; Blakemore, Wolpert, \& Frith, 2002; Karniel, 2002; Wolpert, Miall, \& Kawato, 1998). If the predicted and actually sensed sensory effects coincide, agency is experienced. If they do not coincide, this event is perceived as external, and SoA is not experienced. This predictive feed-forward model or "comparator model" was used to explain the perceptual tuning to stimuli self-generated by subjects (Weiskrantz, Elliott, \& Darlington, 1971) as a possible cause of disruption in the sense of control over their actions in schizophrenia (Blakemore et al., 2002). The theoretical idea of somatosensory cortical activity suppression due to the prediction of one's own motions and the accompanying sensory effects has been confirmed in some studies: behavioral (Weiskrantz et al., 1971; Bays, Wolpert, \& Flanagan, 2005; Blakemore, Frith, \& Wolpert, 1999), MEG (Hesse, Nishitani, Fink, Jousmäki, \& Hari, 2010), fMRI (Blakemore, Wolpert, \& Frith, 1998; Blakemore et al., 2000; Shergill, White, Joyce, Bays, Wolpert, \& Frith, 2013), EEG and ERPs (Abbruzzese, Ratto, Favale, \& Abbruzzese, 1981; Bernier, Burle, Vidal, Hasbroucq, \& Blouin, 2009; Benazet, Thénault, Whittingstall, \& Bernier, 2016; Sidarus, Vuorre, \& Haggard, 2017).

Other approaches emphasize the role of prejudice and external situation signals for maintaining SoA (postdictive model). It has been shown that the priming of subjects with a prejudice relevant to the movement that is actually performed by another person leads to an evaluation of the action as one's own. For example, Wegner and Wheatley (1999) evoked a false SoA for movements that subjects did not do. The significance of environmental signals for SoA was demonstrated as well (Wegner, Sparrow, \& Winerman, 2004). Later it was shown that the priming effect is significant for active voluntary movements (Gentsch \& Schütz-Bosbach, 2011) and is particularly pronounced for passive involuntary movements (Moore, Wegner, \& Haggard, 2009). A dependence of SoA on the subject's conviction about the existence of causal relationships between intention and an external event was also shown (Desantis, Roussel, \& Waszak, 2011). This conforms well with the vast literature about the role of postdictive phenomena in various cognitive processes (for a review, see Shimojo, 2014).

It is possible to assume that both approaches are relevant to real agency mechanisms, a case made, for example, by Kumar \& Srinivasan $(2014,2017)$, who examined the dependence of the sense of agency on the hierarchy of the management level (the upper level of the goal and the lower perceptual-motor level). Some researchers believe that in the context of SoA there is a clear distinction between the feeling of agency (FoA) and the judgment of agency (JoA) (Gallagher, 2000, 2006; Synofzik, Vosgerau, \& Newen, 2008; Bayne \& Pacherie, 2007). According to these authors, FoA is a low-level experience of being the agent of an action, without ex- 
plicitly thinking about the action, whereas JoA relies on a conceptual, interpreting judgment about the authorship of an action, based on the notion of an apparent link between action and result. It was emphasized that these two aspects of agency may not in fact be linked. For example, an unexpected consequence of an action may not cause FoA, but both action and result may be referred to in JoA, if the prejudice and context suggest such a connection. It is believed that JoA is not associated with the sensorimotor system, but is a higher-level process of causal attribution. A good overview of the experimental paradigms and theoretical concepts of the sense of agency can be found in Moore (2016). A sense of ownership (SO) should also be noted in this context. It is a pre-reflexive feeling that "my body is moving", regardless of whether this movement is active (voluntary) or passive (performed by another person or device) (Tsakiris, Schütz-Bosbach, \& Gallagher, 2007).

Despite numerous empirical and theoretical studies, some issues require clarification. Is the sense of agency related to the physical possibility of performing a certain action? In particular, would a passive movement in response to a target stimulus be accompanied by a sense of agency if such a movement occurred within a response time that could not be actively reproduced by the subject? Is there a time delay threshold for a passive movement relative to the target stimulus that the movement cannot be perceived as an own one' before the delay (the passive movement's "reaction time") exceeded that threshold? Under which conditions would the sensory brain activity related to passive movements decrease, and would such a decrease be accompanied by an enhanced sense of agency?

To answer these questions, we developed an experimental paradigm that allows us to monitor the parameters of a simple movement by the participant, and simulate it using a servo with a different, precisely defined delay. An experimental study was conducted using this new paradigm.

\section{Method}

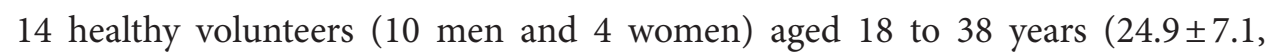
$\mathrm{M} \pm \mathrm{SD}$ ) took part in the experiment. All had normal or corrected-to-normal vision and were naive as to the hypothesis under investigation. All participants were introduced to the procedure and the instructions in writing and agreed to participate in the experiment. The study was consistent with the ethical standards of the Kurchatov complex of NBICS technologies and was performed according to the Helsinki Declaration (1964).

A simple motor action in response to a visual stimulus was the basic paradigm of our study. However, in all experimental conditions except the first, a physical movement was replaced by an imaginary one, and a servo-drive performed the necessary motion. The palm of the right hand was placed on the mounting platform (Fig. 1), and the index finger was fixed in a metal holder consisting of two halves connected by elastic material. The holder blocked all movements of the finger joints except for the metacarpophalangeal joint. Under the holder was a copper plate for the detection of finger lift by the contact method. The translational movement of the holder was transformed into a rotary motion by means of a flexible rod, which was affixed from one side to the bottom of the holder, and on the other side to the shaft of a potentiometer. The holder could be moved by the participant himself by 
raising his finger, or by a digital servo, whose beam transmitted the force of rotation of the servo shaft to the finger through a thread affixed to the upper distal part of the holder. $2 \mathrm{~cm}$ to the left of the holder, a red light emitting diode (LED) was placed at the platform height. Flashing of the LED was used as the target stimulus. The servo drive was covered with a white opaque case, to exclude any distraction that could be caused by its operation. Sensor data recording, LED flashing, and servo drive control were carried out by a computer $(P C)$ using a special program written in the Delphi 2010 environment that communicated with the hardware through a special driver (Fig. 1).

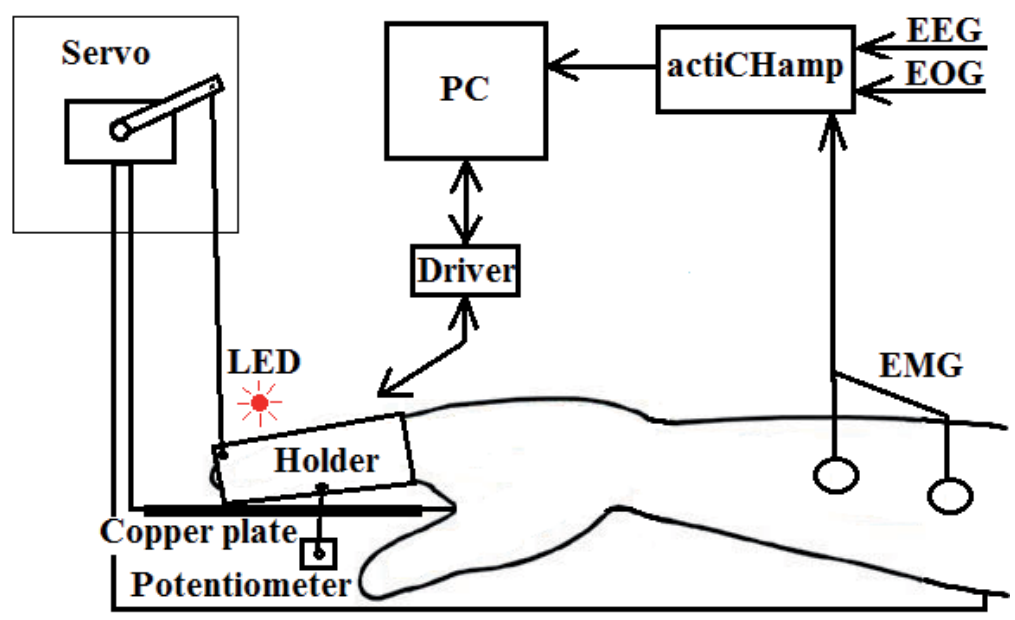

Figure 1. The experimental setup

The experiment included 6 different conditions, which were presented successively, with a 10-15 minute rest break after the 3rd condition.

Active response to a targeted stimulus without a servo (Active Movements - Act$v M o v)$. Participants were asked to carefully look at the LED and lift their finger as soon as possible when it is flashed.

Random triggering of the servo independently of the target stimulus (Stimulus Independent Passive Movements - StIndepPasMov). Participants were asked to carefully look at the LED, but instead of reacting to its flashing, they had to relax their hand as much as possible. The servo drive raised the finger randomly within the test interval, regardless of LED flashing. The distribution of servo operation events is shown in Fig. 2 (the second histogram in the upper row).

Self-raising of the finger in the holder by reacting with a free hand in response to the target stimulus (Delegated Movements - DelegatedMov). In this condition, participants had to react as quickly as possible to LED flashing by pressing the "space" key on the keyboard with their left hand, therefore initiating the servo-drive-mediated raising of the finger. The participant was asked to focus on the LED and the finger in the holder, not the one that presses the key.

Gradual decrease in the delay between the target stimulus and the operation of the servo drive (Passive Movements with Gradual Decrease - PasMovWGrdDec). The participant was asked, in response to each lighting of the LED, to imagine the finger 
rising movement as quickly as possible. The actual finger lifting was performed by the servo at time intervals which were gradually reduced during the experiment. For all participants, the starting delay was $230 \mathrm{~ms}$ from onset of the LED lighting. The delay was decreased by 5 ms every second trial.

A constant small delay between the target stimulus and the activation of the servo drive (Passive Movements with Low Delay - PasMovWLDelay). The participant had to imagine the finger movement in response to the lighting of the LED. The actual movement was carried out by the servo. The servo trip delay was $9 \pm 2 \mathrm{~ms}$ $(\mathrm{M} \pm \mathrm{SD})$.

Test with random operation of the servo drive with reference to the target stimulus (Stimulus Associated Passive Movements - StAssocPasMov). In this condition, the participant was asked to carefully look at the LED but not react to its flashing. The
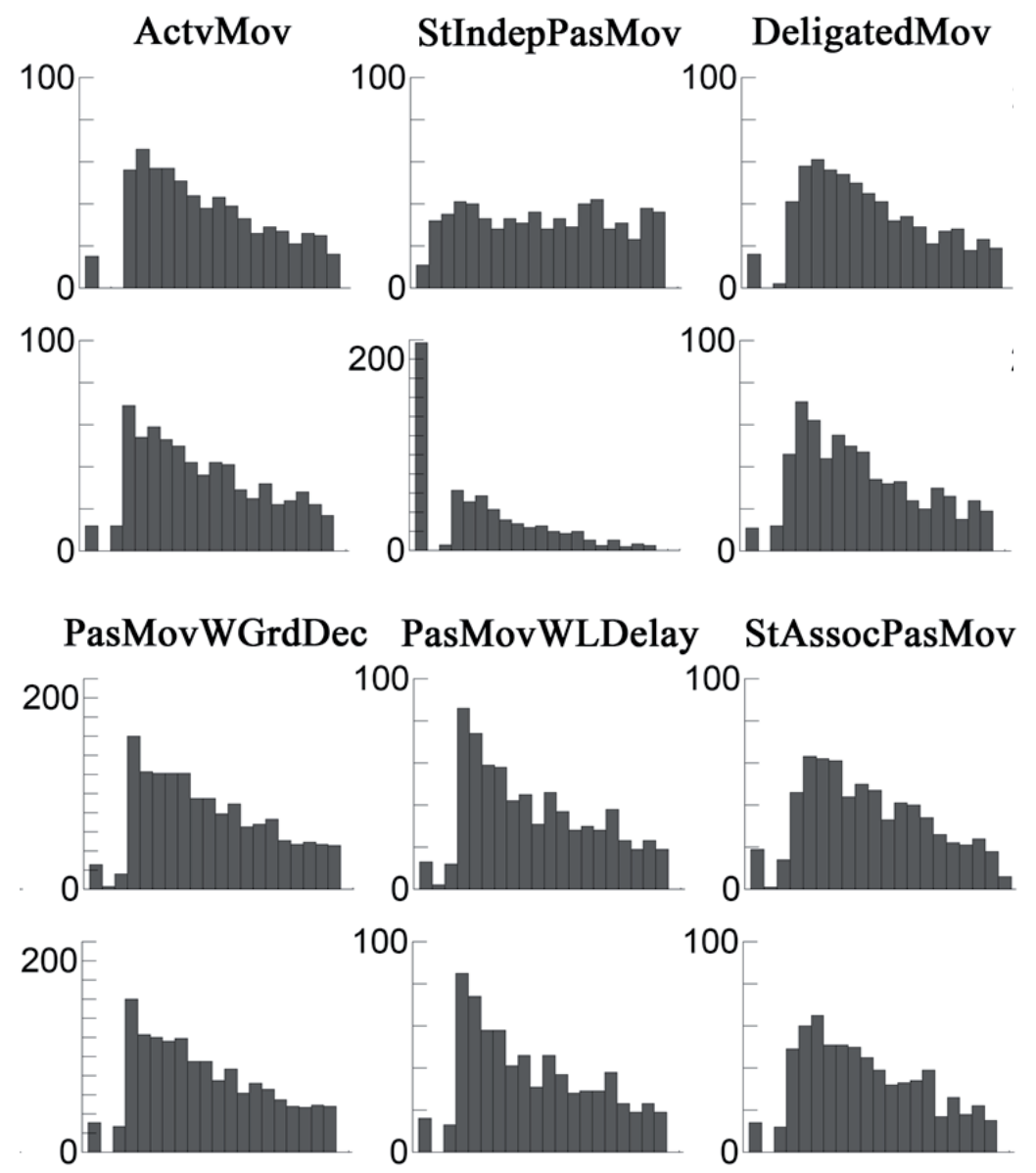

Figure 2. Histograms of the distribution of event time relative to the beginning of intervals for all six test conditions in order, from left to right. The upper row shows servo-tripping events; the bottom row shows LED flashing events. Data are collapsed over the group. X scale is $-350 \mathrm{~ms} \ldots+15000 \mathrm{~ms}$ 
servo was triggered at random intervals, but mostly after the flashing of the LED, and at times a little earlier. For 665 events, the delay time relative to the ignition of the LED was $281 \pm 733 \mathrm{~ms}$ ( $\mathrm{min}-101 \mathrm{~ms}$, max $5149 \mathrm{~ms}$. The distribution of the actuator trip events relative to the beginning of the intervals is shown in Fig. 2 (the rightmost histogram in the upper row).

The distribution of LED flashing under all conditions was set in such a way as to compensate for the increase in the probability of the event over time, which is inevitable when using a uniform distribution for the event generation. Stimulus time distributions for all test conditions are shown in the bottom row of Fig. 2 . The probability of servo events in the StIndepPasMov condition was closer to a uniform distribution. A peak on the histogram for this condition was caused by servo-drivetriggering events that occurred 2 or more seconds before the planned moment of LED lighting (217 out of 648 cases, 33.5\%), which led to the completion of the current interval in the absence of the stimulus. The remaining observations were distributed in the interval from -2203 to $+11754 \mathrm{~ms}$.

At the end of each interval, in all test conditions except for the first one (ActvMov), participants had to report verbally to the experimenter their agency score of the JoA type. A 9-point Likert-type scale (Likert, 1932) had to be used (see Table 1). The scale explanations in the table were made available to the participant on a computer screen, although, after some practice, they did not need to consult with the table in most of the trials. The experimenter noted the scores of each answer in the program.

Table 1. A psychometric scale for the judgment of agency (JoA)

\begin{tabular}{cl}
\hline Rating & \multicolumn{1}{c}{ Explanation } \\
\hline 0 & $\begin{array}{l}\text { You did not even plan the action, there was a clear sense of an external, alien event. } \\
\text { You could perform the action, but its mental representation did not coincide at all } \\
\text { with the actual event and was distinctly felt as not done by you. }\end{array}$ \\
$2-3 \quad \begin{array}{l}\text { You could perform the action, but the event actually observed largely did not coin- } \\
\text { cide with its mental representation; there was a very weak sense of action ownership. }\end{array}$ \\
3-4 $\begin{array}{l}\text { You could perform the action, but the actual observed event did not tangibly coincide } \\
\text { with its mental representation. Some sense of action ownership. }\end{array}$ \\
$5-6 \quad \begin{array}{l}\text { You could perform the action, but the actually observed event slightly mismatched its } \\
\text { mental representation; there was a pronounced sense of action ownership. }\end{array}$ \\
$\begin{array}{l}\text { You could perform the action and the actually observed event was almost synchro- } \\
\text { nous with its mental representation (a feeling of slight disagreement); you experi- } \\
\text { enced a strong sense of action ownership. } \\
\text { You could perform the action and the actually observed event completely coincided } \\
\text { with its mental representation (a feeling of complete agreement); you experienced a } \\
\text { very strong feeling of action ownership. }\end{array}$ \\
\hline
\end{tabular}

Under all test conditions, the LED was flashed randomly within an interval of $15 \mathrm{sec}$ duration (one trial) for a time equal to $100 \mathrm{~ms}$. The beginning of each interval was preceded by a short "tick" warning sound. The sound was presented 
from two speakers on the table in front of the subject at a distance of about a meter, symmetrically on the left and right sides. $200 \mathrm{~ms}$ after the start of either active or passive movement, a confirmation sound was heard, in the form of a consonant or dissonant chord. In the test condition with active motion, the consonant chord sounded if subjects could lift their finger within $350 \mathrm{~ms}$ from the stimulus onset; otherwise, the dissonant chord was presented. In all other test conditions, the consonance chord was always used as the confirmation sound.

In the conditions requiring verbal reports from the subject, $2 \mathrm{sec}$ after the start of the movement there was a short pause, when the experimenter recorded the score for the trial. Each condition lasted 9 min, except for the test with a gradual decrease in the delay between the target stimulus and the servo drive (PasMovWGrdDec), which lasted 15 min.

The logic underlying the order of the experimental conditions was as follows. The condition for a "physical" response to a target stimulus without a servo drive $(A c t v M o v)$ was intended for getting used to the experimental conditions, and for measuring the speed and amplitude of the finger response motion. Mean values of amplitude and speed obtained during this test were used as servo parameters throughout the rest of the experiment.

StIndepPasMov and DelegatedMov conditions served for subjective attribution of the sense of agency to the rating scale in extreme situations, in the absence of motivation for action and unpredictability of the finger-raising events (StIndepPas$M o v)$. An active response to the target stimulus was combined here with imagining of the passive hand's finger movement, which was actually performed by the servo.

PasMovWGrdDec condition was designed to explore the effects of the passive movement's time delay on agency scores and ERP amplitude.

PasMovWLDelay condition was designed to test how the passive movements are perceived, with a low fixed delay between the target stimulus and the servo event.

Finally, StAssocPasMov was used as a control condition. The average delay of servo events in this condition was acceptable for perceiving the target stimulus and imagining the movement in response to it, but, because of the randomness of the passive movement, the participants could not accurately predict when it would start.

After the presentation of each test condition, participants were interviewed to clarify their feelings with regard to the experimental situation.

The EEG was recorded with an actiCHamp amplifier (BrainProducts, Germany). We used 28 electrodes with a common averaged ear reference: Fp1, F7, F3, Fz, FC5, FC1, T7, C3, Cz, CP5, CP1, P7, P3, O1, Oz, FP2, F4, FC6, FC2, FCz, T8, C4, $\mathrm{CP} 6, \mathrm{CP} 2, \mathrm{P} 8, \mathrm{P} 4, \mathrm{Pz}, \mathrm{O} 2$. A vertical and horizontal electrooculogram (EOG) was recorded, as well as an electromyogram (EMG) on the right arm, where a pair of EMG electrodes was placed above the m.extensor at a distance of $3 \mathrm{~cm}$ from each other. The sampling rate was $1000 \mathrm{~Hz}$. EEG and EOG were acquired in the band $0.01 \mathrm{~Hz}-50 \mathrm{~Hz}$, and EMG in the band $5 \mathrm{~Hz}-500 \mathrm{~Hz}$. The recording was made with a notch online filter $50 \mathrm{~Hz}$. The electrode impedance was maintained below $10 \mathrm{k} \Omega$. 
The electrophysiological data were processed with the EEGLAB v13.6.5b package in the Matlab 2013b environment (MathWorks, USA). Statistical analysis was performed using the Statistica 10 package (StatSoft Inc., USA).

\section{Results}

\section{Behavioral results}

All participants felt that there was no feeling of agency (FoA) while raising their finger in all tests except ActvMov and DelegatedMov. In the DelegatedMov condition, some experience of FoA of servo events appeared when the participants were told to refrain from paying attention to the hand performing the physical movement. A sense of ownership (SO), according to self-reports, was always experienced, under all test conditions.

The grand mean response time in the first test condition (ActvMov) was $289 \pm 30 \mathrm{~ms}$. The results for the JoA scale in the next two conditions were, as expected, low for StIndepPasMov (1.4 \pm 0.8 ) and high for DelegatedMov (8.5 \pm 1$)$ (Fig. $4)$. In the condition with a gradual decrease in the delay between the target stimulus and the inclusion of the servo drive (PasMovWGrdDec), no steep changes in agency evaluations were observed, even when the servo event began to lead the LED flashes (see Fig. 3). The minimum and maximum values for group averaged agency ratings, smoothed by a moving average of 6 points, were 5.2 and 6.6, respectively, corresponding to the average delay of the passive movement start of 40 $\mathrm{ms}$ and $99 \mathrm{~ms}$.

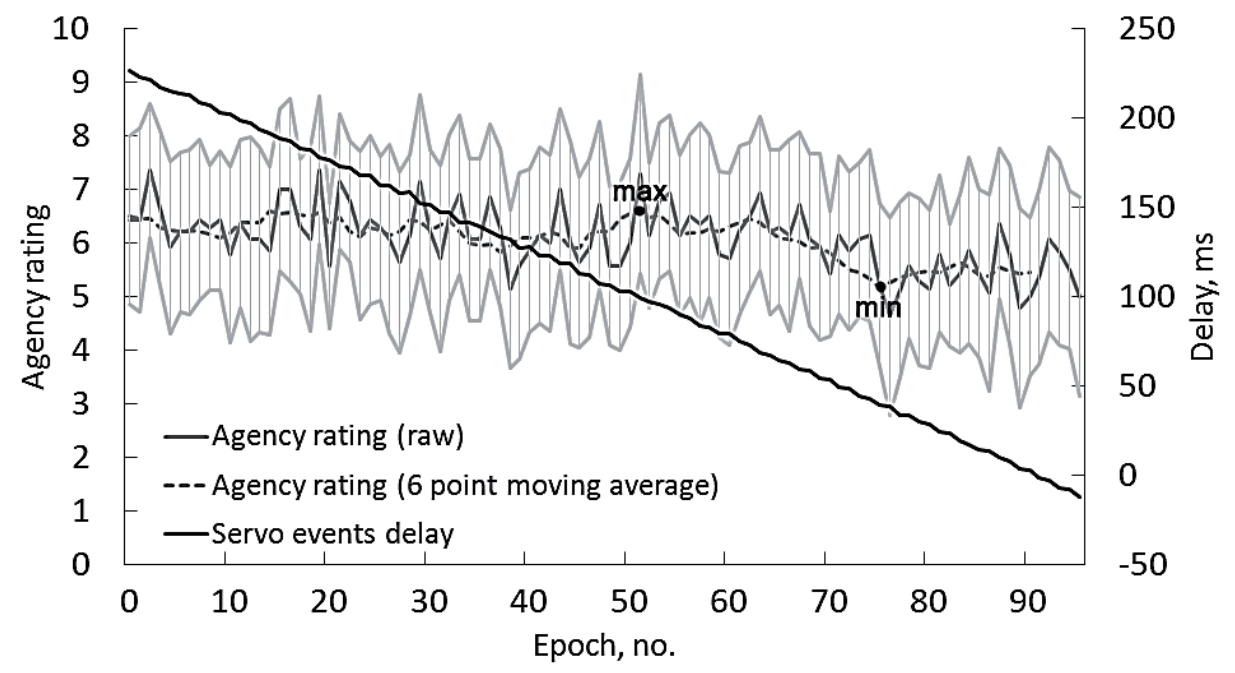

Figure 3. Grand average data of the test condition PasMovWGrdDec. Delays between the onset of the visual stimulus and the raising of the finger by the servo (black lines, right axis) and the agency scores (gray lines, left axis). The horizontal axis presents 96 sequential trials averaged over the group. Light gray lines show a $95 \%$ confidence interval for the agency scores. 
The mean group scores and confidence intervals for four conditions are shown in Fig. 4. The effect of the condition factor was significant (Wilks' $\lambda=0.15$, $\mathrm{F}(3.11)=72.97, \mathrm{p}<0.0001)$. Post-hoc analysis (Fisher LSD) showed the significance of differences for all pairs of conditions $(\mathrm{p}<0.0001)$, except for the pair PasMovWLDelay and StAssocPasMov $(\mathrm{p}=0.81)$. The average group values of the scores of agency scale in the pair of conditions PasMovWLDelay and StAssocPasMov were very close: $5.98 \pm 1.02$ and $5.87 \pm 0.73$. These values, according to the scale of agency used in the study, point to "a pronounced sense of action ownership." The condition with a gradual decrease in the delay between the target stimulus and the activation of the servo (PasMovWGrdDec) was excluded from the inter-test comparative analysis, as its parameters differed too much from the other conditions.

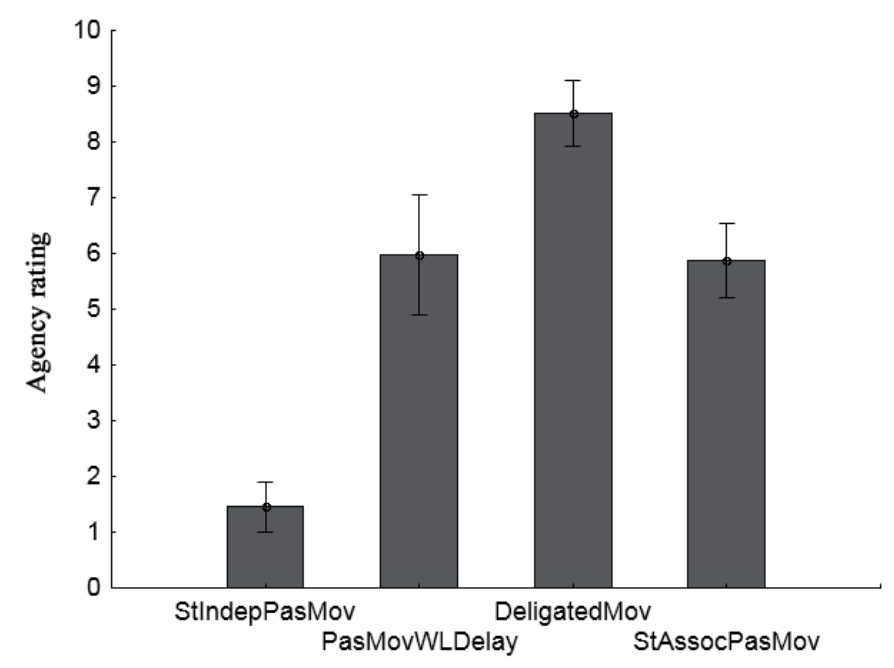

Figure 4. Average scores of the agency scale for the group (vertical scale) for test conditions (horizontal scale). Vertical lines denote $95 \%$ confidence intervals.

\section{Electrophysiological results}

The EEG and EOG data were preprocessed in the following order: filtering with FIR filter (filter order 6601) in the band $0.5 \mathrm{~Hz}-20 \mathrm{~Hz}$; extraction of epochs; baseline correction $(-1000 \mathrm{~ms}$ to $-500 \mathrm{~ms})$; removal of artifact epochs using the EEGLAB function pop_autorej with default parameters (in particular, rejection of all epochs with an amplitude exceeding $\pm 100 \mu \mathrm{V}$ ) with subsequent visual inspection and additional manual rejection. For the EMG, only baseline correction and artifacts rejection were performed.

In most of the conditions, a prominent ERP negative component was observed. The maximum of this component was located at FC1 (contralateral to the involved limb), where its latency was about $115 \mathrm{~ms}$. The ERP topographical scalp maps for different conditions are shown in Fig. 5 (the number of individual averages in each condition was about 40-45 epochs per participant). Based on its latency and topography, this component was identified as the somatosensory $\mathrm{N} 1$.

For further analysis, we chose the region of interest (ROI), for which the maximum severity of the negative somatosensory component of ERPs was observed 
(the ROI is marked with an oval on the map at the far right in Fig. 5). In the group analysis of the ROI, averaged data for all test conditions at mean inter-peak amplitudes were calculated as the difference between the peak amplitude of the negative ERP components (latency about $115 \mathrm{~ms}$ ) and positive ERP components (latency about $200 \mathrm{~ms}$ ), measured as the minimum in the 50-200 ms interval and the maximum in the 100-300 ms interval, respectively.

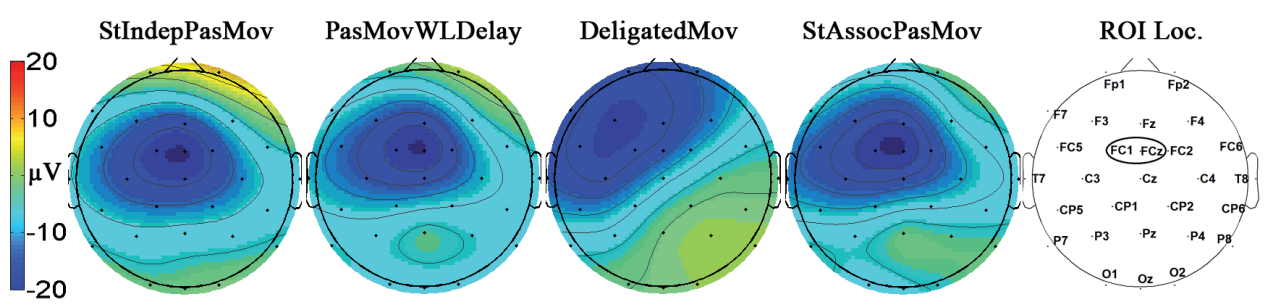

Figure 5. Topographical scalp maps of the grand average ERPs at a time point corresponding to the maximum of the somatosensory component $(115 \mathrm{~ms})$. Electrode positions used in the experiment are shown on a scalp map on the right. The oval on the map marks the region of interest (ROI).

In the test condition PasMovWGrdDec, the peak-to-peak amplitudes were calculated separately for each of the 96 EEG epochs corresponding to the monotonically decreasing servo event delays, group-averaged and smoothed with a 6 point moving average (Fig. 6). The amplitude showed a monotonic increase over most of the delay values, with a maximum delay $(59 \mu \mathrm{V})$ at $49 \mathrm{~ms}$ and a minimum delay $(41 \mu \mathrm{V})$ at $208 \mathrm{~ms}$.

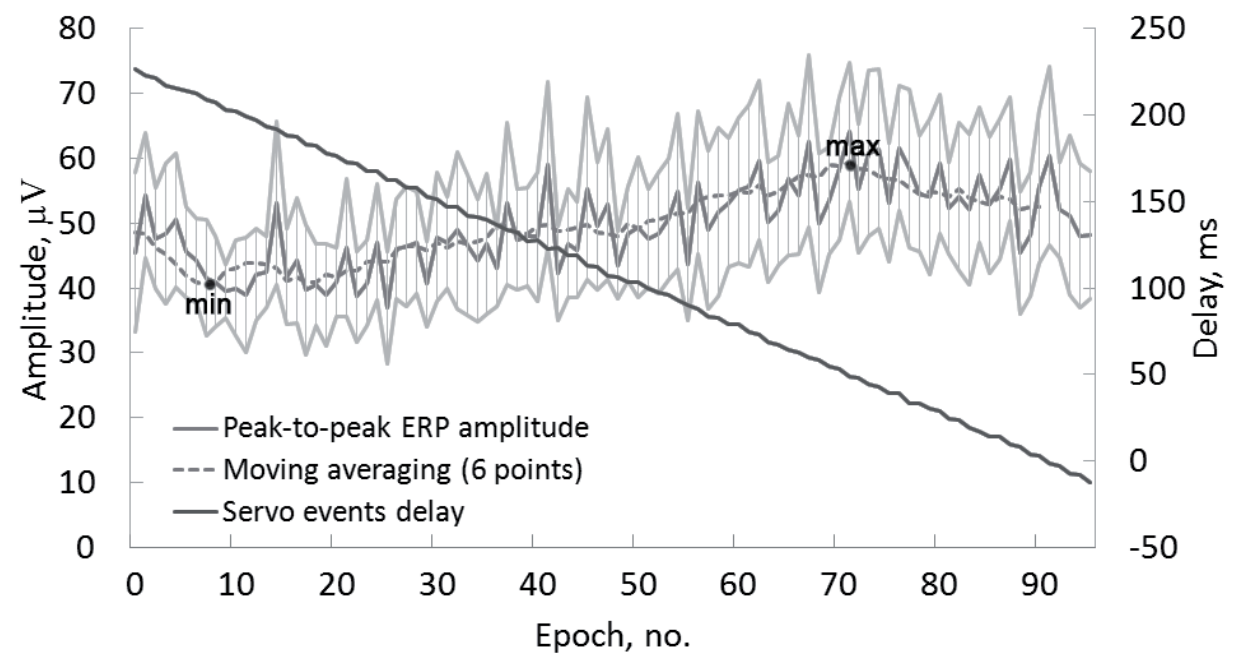

Figure 6. Group average data of the test condition PasMovWGrdDec. Delays between the visual stimulus onset and the raising of the finger by the servo (black lines, right axis) and the peak-to-peak amplitudes of ERP by ROI (gray lines, left axis). The horizontal axis presents 96 sequential trials averaged over the group. Light gray lines show a 95\% confidence interval for the peak-to-peak amplitudes. 
ROI averaged group evoked responses for the remaining five test conditions are presented in Fig. 7A. The ERP waveforms in test ActvMov condition was very different from the others, which was expected due to the fundamental difference in this condition - with physical movement, as against imaginary movement in the other cases. Fig. 7B shows the averaged EMG responses for the same test conditions. Note that the EMG activity amplitude for the ActvMov condition was noticeably different from the others.

EMG amplitude was much lower (almost absent) in all the other conditions, confirming that participants followed instructions and refrained from actively making movements when all that was required was to imagine an active role in making the movement.

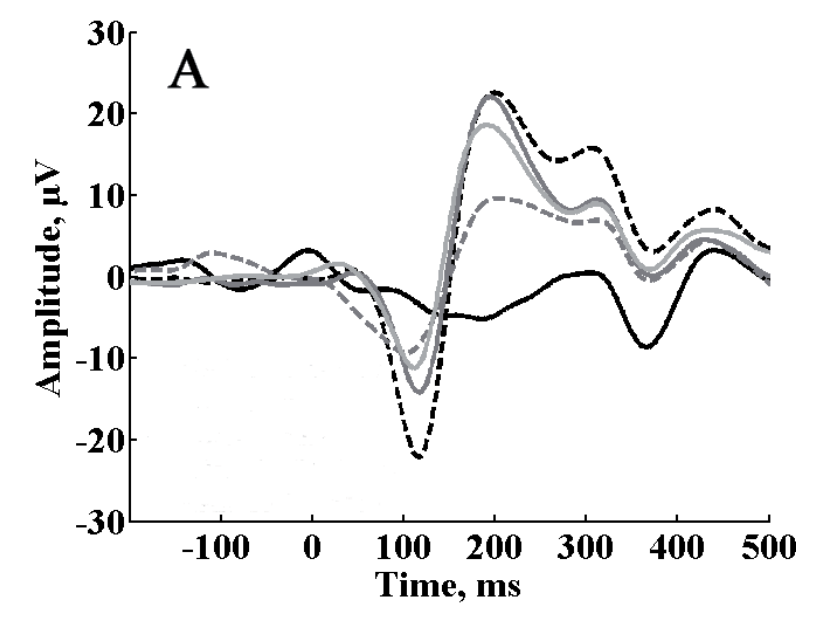

$$
\text { ---StIndepPasMov - ActvMov ---DeligatedMov }
$$

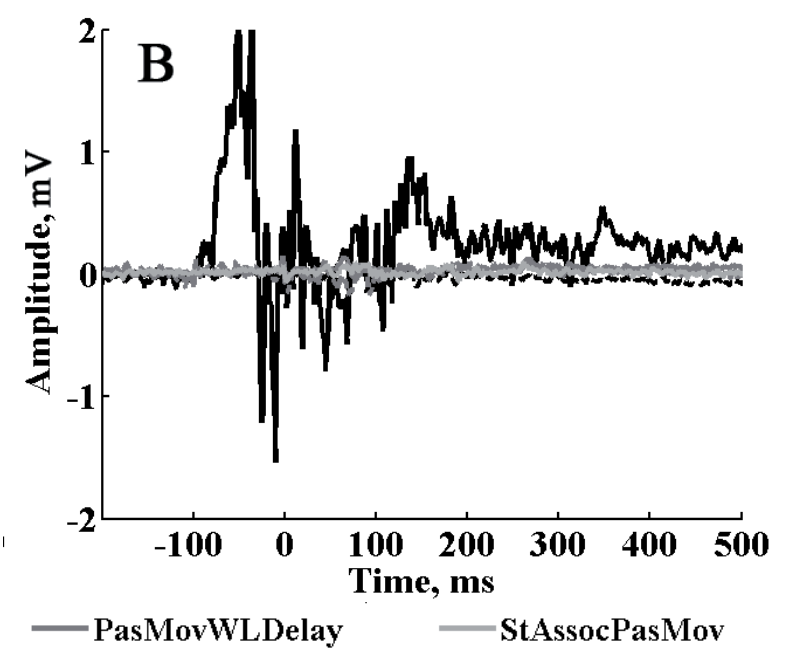

Figure 7. A: Group average ERP for ROI for different test conditions; B: Group average EMG potentials 
The mean values and the confidence intervals of the ERP somatosensory component peak-to-peak amplitude in different conditions are shown in Fig. 8.

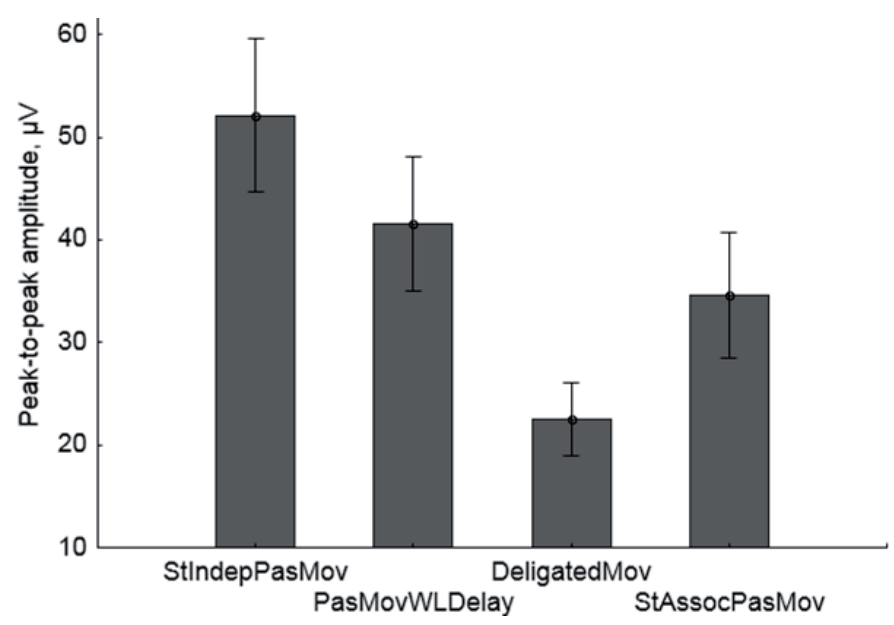

Figure 8. Grand average values of the peak-to-peak amplitude of the ERP somatosensory component (vertical scale) for passive movement conditions. Vertical lines denote 95\% confidence intervals.

ANOVA with repeated measures applied to the peak-to-peak amplitude of the ERP somatosensory component showed significant effect of the condition factor (Wilks' $\lambda=0.12, F(3,11)=26.40, p<0.0001$ ). According to the post-hoc test (Fisher LSD), all pairwise differences between the conditions were statistically significant $(\mathrm{p}=0.013$ for the pair PasMovWLDelay - StAssocPasMov and $\mathrm{p}<0.0001$ for the other five pairs).

In the passive movement conditions with imagining an active role in making the movement, the group average data for agency scores (Fig. 4) and peak-to-peak ERP amplitudes (Fig. 8) appeared to be inversely correlated: the higher the agency score in a condition, the less the peak-to-peak ERP. An ANCOVA applied to the peak-to-peak ERP amplitude as the dependent variable, with the conditions as a categorical predictor and the agency scores as a continuous predictor, showed only the influence of the condition factor $(\mathrm{F}(3,51)=4.53, \mathrm{p}<0.0069)$, while the agency score factor effect was not significant $(\mathrm{p}<0.6798)$.

In group averaged and smoothed-over trials, peak-to-peak ERP amplitudes (gray graph in Fig. 6) and agent scores (gray graph in Fig. 3) are slightly correlated within the PasMovWGrdDec condition (Spearman's $\mathrm{R}=-0.29 ; \mathrm{p}=0.003$ ). This could reflect the influence of the common factor of the passive movement delay, which correlated negatively with the ERP amplitude (Spearman's $R=-0.68, \mathrm{p}<0.001$ ) and positively with the agency scores (Spearman's $\mathrm{R}=0.49, \mathrm{p}<0.001$ ).

\section{Discussion}

We investigated SoA in a group of healthy participants, asking them to imagine that they were actively moving their finger, although actually it was moved by a mechanical device, with a different time delay relative to a simple visual stimulus. One 
of our assumptions was that under these conditions the participants would associate the passive movements with their own intention, perceiving the actual movement as actively made by them, even if it was performed earlier than their earliest possible reaction time. Contrary to this assumption and in accordance with the survey results, in all test conditions with passive movement there was no apparent FoA. However, the participants' responses corresponded to a perception that these actions could have been performed by them; moreover, they typically chose scores related to the statement that the action only "slightly mismatched its mental representation" (except for the StIndepPasMov condition, in which there was no sense of agency). The sense of ownership (SO) was experienced in all test conditions. This confirms the idea that the SoA for any movement includes the SO as its most basic aspect (Gallagher, 2000), which can be also accompanied by two further aspects, FoA and JoA (Synofzik, Vosgerau, \& Newen, 2008; Bayne \& Pacherie, 2007).

In the PasMovWGrdDec and PasMovWLDelay conditions, the scores did not reduce to the minimum values even when it was physically impossible to perform the action that was executed by a servo, because the delay between the target stimulus and the passive movement was too small. Interestingly, no abrupt changes were observed in the group average of agency scores or in their individual dynamics that might be related to a threshold time given from the stimulus such that the action cannot be perceived as fully "owned" if it appears earlier than the threshold time. Assuming that the scores of the scale we used really reflected the JoA phenomenon, it turns out that this aspect of SoA persists with any delay between the target stimulus and the action.

The somatosensory ERP complex observed in this study apparently did not differ from a typical response to tactile stimulation (see, for example, Eimer, Maravita, Van Velzen, Husain, \& Driver, 2002). It was pronounced under passive movement conditions and absent in the active movement condition. In all conditions when the passive movement was expected, the amplitude of the ERP somatosensory component decreased, possibly as the result of the suppression of somatosensory brain activity. JoA was inversely related to the magnitude of this activity. For example, with a gradual decrease in the delay in the PasMovWGrdDec condition, agency scores decreased along with an increase in somatosensory ERP complex peak-topeak amplitude. Thus, active movement, executed with muscle activity, seemed to be not so important for JoA, as compared to compliance with expectations: this is what really mattered.

The results obtained on the whole allow us to conclude that peak-to-peak amplitude of somatosensory ERP reflects the unexpectedness of the passive movement event quite well. At the same time, it turned out that the scores on the scale of agency effectively differentiate the extreme conditions for predictability of events: with self-generation of the servo event by the free hand (DelegatedMov) and in the condition of random triggering of the servo, regardless of the target stimulus (StIndepPasMov). Meanwhile, indistinguishability was observed in the points of agency for the conditions of PasMovWLDelay and StAssocPasMov. In the PasMovWGrdDec condition, even with a negative delay of $-12 \pm 2.4 \mathrm{~ms}(\mathrm{M} \pm \mathrm{SD})$, the average score was $5 \pm 1.85(\mathrm{M} \pm \mathrm{SD})$.

The peak-to-peak amplitude of the somatosensory ERP was greater in the condition with a constant low delay (PasMovWLDelay), compared to the condition 
of random delay associated with the stimulus (StAssocPasMov). It could turn out that the constant but very small $(9 \pm 2 \mathrm{~ms}(\mathrm{M} \pm \mathrm{SD}))$ passive movement delay in the PasMovWLDelay condition was subjectively similar in its surprise element to the situation of a random variation of the passive movement delay in the StAssocPasMov condition. The variation of the passive movement delay in the StAssocPasMov condition was organized using the same time distribution as for the target stimulus (see Fig. 2). In addition, the passive movement, despite the randomness of the delay, almost always (in $98 \%$ of cases) occurred after the occurrence of the target stimulus.

The behavioral results obtained may be related either to psychophysiological adaptation to new temporary relationships between the targeted stimulus and the intended action, or to insufficient reflection of subjective sensations in the questionnaire's score descriptions, or to the effect of prejudice. The experiment assumed an unambiguous connection between the appearance of the target stimulus and the subsequent action. Such "context pressure" may promote JoA, according to the postdictive model of the influence of prejudice and situational factors. It is tempting to accept as one's own an action that is habitual and necessary, even if it is not felt in this way (no FoA). Probably prejudice had a greater effect on the agency of the motor event than did temporary uncertainty.

It may be impossible to make a correct subjective score-based evaluation of agency under certain conditions, including at least some of those used in our study. Nevertheless, the test conditions in the first part of the experiment, creating situations of high unpredictability (StIndepPasMov) and full control over passive movements (DelegatedMov) showed plausible scores, which indicate, at least, the participants' understanding of the instruction and the adequacy of the scale for its extreme values. Finally, the outcome of relatively high agency scores for the passive movement with negative "reaction time" can be explained as follows. Due to the servo drive's adjustment to individual motion parameters, its movement was not very fast and partially overlapped with the time of the target stimulus. Thus, behavioral assessment in this situation can make a subjective determination of the movement's beginning impossible, although it could in general be recognized as self-initiated, again, in view of the need to mentally respond to the target stimulus.

\section{Conclusions}

The results obtained in this study support the idea of the independence of the sensory (FoA) and the evaluative (JoA) aspects of the agency experience (Gallagher, 2000, 2006; Synofzik et al., 2008). In part, they can be explained using a predictive feed-forward model (Blakemore et al., 1999, 2000, 2002), since in the absence of active motions, the FoA effect was not observed. On the other hand, the negative relationship between the amplitude of somatosensory ERP and the delay of the passive motion, as well as the dependence of the ERP amplitude on the surprise factor of passive motion, allows the following, more elaborated explanation. Prediction of the movement's sensory effects remains possible with passive movements, provided that it is coordinated with the mental representation of the situation. If so, then the feed-forward model is not applicable in this case, since the model requires active implementation of motor commands to construct predictions of sensory conse- 
quences. This argument, together with the presence of JoA in the context of various delays between the target stimulus and the motor event, even when these delays are extremely short, provides crucial evidence in favor of postdictive models of agency experience, in which the leading role is assigned to the situational factors of prejudice and contextual knowledge related to the action (Wegner \& Wheatley, 1999; Wegner et al., 2004). It seems that the "context pressure" of the experimental situation, which presupposes a mandatory response to the stimulus, enables prediction of passive movements and their sensory consequences.

In future studies, the subjective evaluation of the time intervals of the actionresult (e.g., Engbert, Wohlschläger, \& Haggard, 2008) can be used as an alternative and possibly a more sensitive measure than the survey-based agency estimation. This method follows from the "intentional binding" effect discovered by Haggard, Clark, \& Kalogeras, (2002): the interval between the action and the subsequent event is estimated shorter if it is accompanied by a sense of agency.

\section{Acknowledgments}

This work was in part supported by the Russian Science Foundation, grant RScF, project No. 14-28-00234.

\section{References}

Abbruzzese, G., Ratto, S., Favale, E., \& Abbruzzese, M. (1981). Proprioceptive modulation of somatosensory evoked potentials during active or passive finger movements in man. Journal of Neurology, Neurosurgery \& Psychiatry, 44(10), 942-949. doi: 10.1136/jnnp.44.10.942

Bayne, T., \& Pacherie, E. (2007). Narrators and comparators: The architecture of agentive selfawareness. Synthese, 159(3), 475-491. doi: 10.1007/s11229-007-9239-9

Bays, P.M., Wolpert, D.M., \& Flanagan, J.R. (2005). Perception of the consequences of self-action is temporally tuned and event driven. Current Biology, 15(12), 1125-1128. doi: 10.1016/j. cub.2005.05.023

Bernier, P.M., Burle, B., Vidal, F., Hasbroucq, T., \& Blouin, J. (2009). Direct evidence for cortical suppression of somatosensory afferents during visuomotor adaptation. Cerebral Cortex, 19(9), 2106-2113. 10.1093/cercor/bhn233

Benazet, M., Thénault, F., Whittingstall, K., \& Bernier, P. M. (2016). Attenuation of visual reafferent signals in the parietal cortex during voluntary movement. Journal of Neurophysiology, 116(4), 1831-1839. doi: 10.1152/jn.00231.2016

Blakemore, S.J., Wolpert, D.M., \& Frith, C.D. (1998). Central cancellation of self-produced tickle sensation. Nature Neuroscience, 1(7), 635-640. doi: 10.1038/2870

Blakemore, S.J., Frith, C.D., \& Wolpert, D.M. (1999). Spatio-temporal prediction modulates the perception of self-produced stimuli. Journal of Cognitive Neuroscience, 11(5), 551-559. doi: $10.1162 / 089892999563607$

Blakemore, S.J., Wolpert, D.M., \& Frith, C.D. (2000). Why can't you tickle yourself? Neuroreport, 11(11), R11-R16. doi: 10.1097/00001756-200008030-00002

Blakemore, S-J, Wolpert, D. M., \& Frith, C. D. (2002). Abnormalities in the awareness of action. Trends in Cognitive Sciences, 6(6), 237-242. doi: 10.1016/S1364-6613(02)01907-1

Desantis A., Roussel C., \& Waszak F. (2011). On the influence of causal beliefs on the feeling of agency. Consciousness and Cognition, 20, 1211-1220. doi: 10.1016/j.concog.2011.02.012 
Eimer, M., Maravita, A., Van Velzen, J., Husain, M., \& Driver, J. (2002). The electrophysiology of tactile extinction: ERP correlates of unconscious somatosensory processing. Neuropsychologia, 40(13), 2438-2447. doi: 10.1016/S0028-3932(02)00079-9

Engbert, K., Wohlschläger, A., \& Haggard, P. (2008). Who is causing what? The sense of agency is relational and efferent-triggered. Cognition, 107(2), 693-704. doi: 10.1016/j.cognition. 2007.07.021

Gallagher, S. (2000). Philosophical conceptions of the self: Implications for cognitive science. Trends in Cognitive Sciences, 4(1), 14-21. doi: 10.1016/S1364-6613(99)01417-5

Gallagher, S. (2002). Experimenting with introspection. Trends in Cognitive Sciences, 6, 374-375. doi :10.1016/s1364-6613(02)01979-4

Gallagher, S. (2006). How the body shapes the mind. Oxford: Oxford University Press.

Gentsch, A., \& Schütz-Bosbach, S. (2011). I did it: Unconscious expectation of sensory consequences modulates the experience of self-agency and its functional signature. Journal of Cognitive Neuroscience, 23(12), 3817-3828. doi: 10.1162/jocn_a_00012

Haggard, P., Clark, S., \& Kalogeras, J. (2002). Voluntary action and conscious awareness. Nature Neuroscience, 5(4), 382-385. doi: 10.1038/nn827

Hesse, M.D., Nishitani, N., Fink, G.R., Jousmäki, V., \& Hari, R. (2010). Attenuation of somatosensory responses to self-produced tactile stimulation. Cerebral Cortex, 20(2), 425-432. doi: $10.1093 /$ cercor/bhp110

Karniel, A. (2002). Three creatures named 'forward model'. Neural Networks, 15(3), 305-307. doi: 10.1016/S0893-6080(02)00020-5

Likert, R. (1932). A technique for the measurement of attitudes. Archives of Psychology, 22(140), 5-55.

Kumar D., \& Srinivasan N. (2014). Naturalizing sense of agency with a hierarchical event-control approach. PLoS One, 9, e92431. doi: 10.1371/journal.pone.0092431

Kumar, D., \& Srinivasan, N. (2017). Multi-scale control influences sense of agency: Investigating intentional binding using event-control approach. Consciousness and Cognition, 49, 1-14. doi: 10.1016/j.concog.2016.12.014

Moore J.W., Wegner D.M., \& Haggard P. (2009). Modulating the sense of agency with external cues. Consciousness and Cognition, 18, 1056-1064. doi: 10.1016/j.concog.2009.05.004

Moore, J. W. (2016). What is the sense of agency and why does it matter? Frontiers in Psychology, 7. doi: 10.3389 /fpsyg.2016.01272

Shergill, S.S., White, T.P., Joyce, D.W., Bays, P.M., Wolpert, D.M., \& Frith, C.D. (2013). Modulation of somatosensory processing by action. Neuroimage, 70, 356-362. doi: 10.1016/j. neuroimage.2012.12.043

Sidarus, N., Vuorre, M., \& Haggard, P. (2017). How action selection influences the sense of agency: An ERP study. NeuroImage, 150, 1-13. doi: 10.1016/j.neuroimage.2017.02.015

Shimojo, S. (2014). Postdiction: Iits implications on visual awareness, hindsight, and sense of agency. Frontiers in Psychology, 5: 196. doi: 10.3389/fpsyg.2014.00196

Synofzik, M., Vosgerau, G., \& Newen, A. (2008). Beyond the comparator model: A multifactorial two-step account of agency. Consciousness and Cognition, 17(1), 219-239. doi: 10.1016/j. concog.2007.03.010

Tsakiris, M., Schütz-Bosbach, S., \& Gallagher, S. (2007). On agency and body-ownership: phenomenological and neurocognitive reflections. Consciousness and Cognition, 16(3), 645-660. 10.1016/j.concog.2007.05.012

Wegner, D.M. \& Wheatley, T. (1999). Apparent mental causation: Sources of the experience of will. American Psychologist, 54(7), 480-492. doi: 10.1037/0003-066X.54.7.480 
Wegner, D.M., Sparrow, B., \& Winerman, L. (2004). Vicarious agency: Experiencing control over the movements of others. Journal of Personality and Social Psychology, 86(6), 838. doi: 10.1037/0022-3514.86.6.838

Weiskrantz, L., Elliott, J., \& Darlington, C. (1971). Preliminary observations of tickling oneself. Nature, 23, 598-599. doi: 10.1038/230598a0

Wolpert, D.M., Miall, R. C., \& Kawato, M. (1998). Internal models in the cerebellum. Trends in Cognitive Sciences, 2(9), 338-347. doi: 10.1016/S1364-6613(98)01221-2

Original manuscript received June 12, 2017 Revised manuscript accepted July 01, 2017

First published online September 30, 2017 